\title{
Microbiome-driven identification of microbial indicators for postharvest diseases of sugar beets
}

Peter Kusstatscher ${ }^{1,2}$, Christin Zachow ${ }^{1}$, Karsten Harms ${ }^{3}$, Johann Maier ${ }^{3}$, Herbert Eigner ${ }^{4}$, Gabriele Berg ${ }^{2}$ and Tomislav Cernava ${ }^{2,5^{*}}$ (D)

\begin{abstract}
Background: Sugar loss due to storage rot has a substantial economic impact on the sugar industry. The gradual spread of saprophytic fungi such as Fusarium and Penicillium spp. during storage in beet clamps is an ongoing challenge for postharvest processing. Early detection of shifts in microbial communities in beet clamps is a promising approach for the initiation of targeted countermeasures during developing storage rot. In a combined approach, high-throughput sequencing of bacterial and fungal genetic markers was complemented with cultivation-dependent methods and provided detailed insights into microbial communities colonizing stored roots. These data were used to develop a multi-target qPCR technique for early detection of postharvest diseases.

Results: The comparison of beet microbiomes from six clamps in Austria and Germany highlighted regional differences; nevertheless, universal indicators of the health status were identified. Apart from a significant decrease in microbial diversity in decaying sugar beets $(p \leq 0.01)$, a distinctive shift in the taxonomic composition of the overall microbiome was found. Fungal taxa such as Candida and Penicillium together with the gram-positive Lactobacillus were the main disease indicators in the microbiome of decaying sugar beets. In contrast, the genera Plectosphaerella and Vishniacozyma as well as a higher microbial diversity in general were found to reflect the microbiome of healthy beets. Based on these findings, a qPCR-based early detection technique was developed and confirmed a twofold decrease of health indicators and an up to 10,000-fold increase of disease indicators in beet clamps. This was further verified with analyses of the sugar content in storage samples.

Conclusion: By conducting a detailed assessment of temporal microbiome changes during the storage of sugar beets, distinct indicator species were identified that reflect progressing rot and losses in sugar content. The insights generated in this study provide a novel basis to improve current or develop next-generation postharvest management techniques by tracking disease indicators during storage.
\end{abstract}

Keywords: Beta vulgaris, Storage rot, Indicator species, Phytopathogens, Bacterial microbiome, Fungal microbiome

\section{Background}

Plant-colonizing microorganisms live in close relationship with their host and are a crucial factor for plant growth and health [1-3]. For various crop plants, this was observed along the entire value-chain including the postharvest period [4]. The exploration of plant-microbe interactions, plant-beneficial bacteria and fungi including

\footnotetext{
* Correspondence: tomislav.cernava@tugraz.at

${ }^{2}$ Institute of Environmental Biotechnology, Graz University of Technology, Petersgasse 12, 8010 Graz, Austria

${ }^{5}$ Roombiotic GmbH, c/o: SciencePark, Stremayrgasse 16/IV, 8010 Graz, Austria Full list of author information is available at the end of the article
}

yeasts, their functions, and modes of action is a key for advanced developments related to biotechnological applications in agriculture $[2,5]$. However, the development of postharvest applications based on biologicals is challenging due to the great diversity of postharvest pathogens as well as the often highly challenging postharvest treatments and storage conditions $[6,7]$. The herbaceous dicotyledonous plant, Beta vulgaris L. (sugar beet) is the main crop for sugar production (sucrose content up to $18 \%$ ) in temperate regions all over the world [8]. A number of plant pathogens such as Pythium

(c) The Author(s). 2019 Open Access This article is distributed under the terms of the Creative Commons Attribution 4.0 International License (http://creativecommons.org/licenses/by/4.0/), which permits unrestricted use, distribution, and 
ultimum Trow [9], Rhizoctonia solani Kühn [10], and Cercospora beticola Sacc. [11] cause severe harvest shortfalls due to seedling rot or late root rot [12]. After harvest, starting from late October, sugar beets are stored in Europe directly on the fields for a maximum of 60 days due to limited process capacities and increased economic viability of sugar refineries. High water (76\%) and sugar content (18\%) in the unprocessed beets [13] provide perfect conditions for microbial colonization, especially when cracks, root tip breakage, and fresh wounds on the surface provide easy entry points [14]. Microbial colonization, mainly by pathogenic or saprophytic fungi such as Fusarium, Penicillium, and Botrytis spp., leads to substantial sugar yield losses. A major observation is microbial inversion of sucrose into unwanted glucose and fructose molecules [15]. The combined occurrence of microbial degradation, respiration of the beet root, synthesis of raffinose, and other causes can yield sugar losses of up to $50-60 \%$ during storage $[16,17]$.

Natural antagonists that are part of the indigenous beet microbiome, previously studied by Zachow and colleagues (2008) [18], carry the potential for alternative plant protection applications during growth and postharvest $[19,20]$. In our previous study, we found correlations between the disease incidence in sugar beet fields and the antagonistic potential of the prevalent microbiota [21]. These observations provide the basis for sustainable methods to prevent high sugar yield losses, caused by fungal infection with a targeted use of antagonistic microorganisms that could also provide postharvest protection [22]. However, in order to develop targeted and sustainable countermeasures, it is crucial to identify key players in the rot onset and to improve early detection strategies of rot-causing pathogens for beet clamps. Moreover, when biological control is employed, it is important to understand to which natural counterparts beneficial microorganisms will be exposed. Although rot-causing fungal pathogens were previously identified [14], the health-related dynamics of bacteria and fungi in stored sugar beets remained unexplored.

The aim of this study was to analyze temporal community changes in the microbiome of stored roots, correlate them to sugar beet health, and finally integrate the generated knowledge into a novel disease detection technique. Therefore, we investigated the bacterial and fungal microbiome of stored sugar beets in different beet clamps located in important cultivation areas of Austria and Germany. By implementing a detailed assessment of the beet clamp microbiome, specific biological markers indicating disease development in stored beets were found. These observations were thereafter confirmed with sugar beets stored under controlled conditions to verify the applicability of the identified markers. The overall findings provide a basis for novel postharvest management techniques that implement microbial and molecular markers for targeted countermeasures.

\section{Results}

Identification of fungal taxa from decaying sugar beets

In order to identify fungal taxa in infected sugar beets from clamps in Austria and Germany, two complementary methods were applied. The community structure was reconstructed with Sanger sequencing of $18 \mathrm{~S}$ rRNA gene fragments from fungal isolates and ITS Illumina amplicon sequencing of total community DNA (Fig. 1). The 18S rRNA gene sequencing-based community reconstruction with 120 fungal strains indicated a fungal

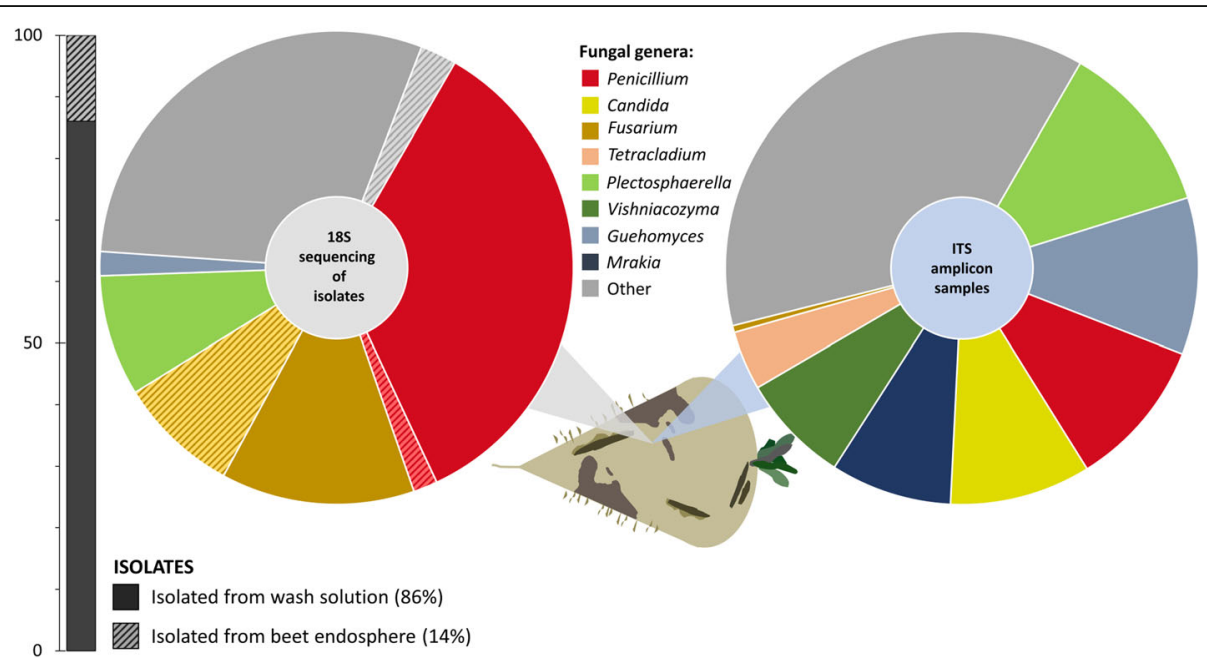

Fig. 1 Comparison of $18 \mathrm{~S}$ rRNA gene fragment sequencing results of fungal strains isolated from beet clamps in Austria and Germany and ITS sequencing of amplicon samples. The obtained sequences were assigned up to genus level. Color-coded segments indicate different genera in both datasets. Shaded areas represent the fraction of fungal isolates obtained from the beet endosphere 
community structure with 11 different genera, which was dominated by Penicillium (37\%) and Fusarium (22\%) species, while ITS amplicon sequencing indicated a more diverse composition. A total of 80 amplicon datasets revealed more than 50 different fungal genera. The most prominent genera were assigned to Plectosphaerella (11\%), Guehomyces (10\%), Penicillium (10\%), Candida (10\%), Mrakia (8\%), Vishniacozyma (8\%), and Tetracladium (4\%). While Penicillium was abundant in both approaches, Fusarium was only predominant in the isolate-based community reconstruction. Moreover, the highest proportion of fungal strains $(86 \%)$ was recovered from the beet surface; however, a substantial fraction of the identified Fusarium species (39\%) originated from the sugar beet endosphere.

\section{Microbial diversity was significantly decreased in decaying sugar beets}

The comparison of amplicon data obtained from 120 samples of healthy and decaying sugar beets showed a significantly lower bacterial diversity in infected samples (Shannon index: 4.5 (16S) and 3.5 (ITS)) compared to the microbiome of healthy sugar beets (Shannon index 5.5 (16S) and 4.5 (ITS)) (Fig. 2b). The calculated BrayCurtis distances showed significant differences in the composition of the microbiomes of the two groups. When a group-wise comparison was conducted, samples of decaying sugar beets $(n=80)$ clustered significantly
( $p$ value $\leq 0.01)$ different from samples of healthy sugar beets $(n=40)$. The variation within the infected group was found to be higher, compared to the healthy samples, which clustered more closely together (Fig. 2a).

\section{The core microbiome composition was altered in decaying sugar beets}

Taxonomic assignments of the identified features indicated a decay-specific microbiome of the analyzed sugar beets. The comparison of healthy and decaying samples showed a clearly distinguishable composition of taxa in both bacterial and fungal amplicon reads. Proteobacteria with an average relative abundance of $41 \%$ (healthy samples) and 51\% (decaying samples) were the most abundant taxa on phylum level. Bacteriodetes $(27 \%$ and $12.5 \%)$ and Actinobacteria (28\% and $11 \%$ ) were also highly abundant in both groups. The main difference between both groups was due to the phylum Firmicutes ( $0.4 \%$ in healthy and $25 \%$ in decaying samples). A major fraction of Firmicutes in the decaying samples belonged to the order of Lactobacillales (24\%). The predominant Proteobaceria in healthy samples were mainly members of the orders Pseudomonadales (10\%), Sphingomonadales (9\%), Rhizobiales (8.5\%), Xanthomonadales (6.5\%), and Enterobacteriales (2.5\%). In contrast, the 51\% Proteobacteria found in decaying samples belonged to the orders Rhodospirillales (20\%), Enterobacteriales (8\%), Pseudomonadales (8\%), Xanthomonadales (5\%),

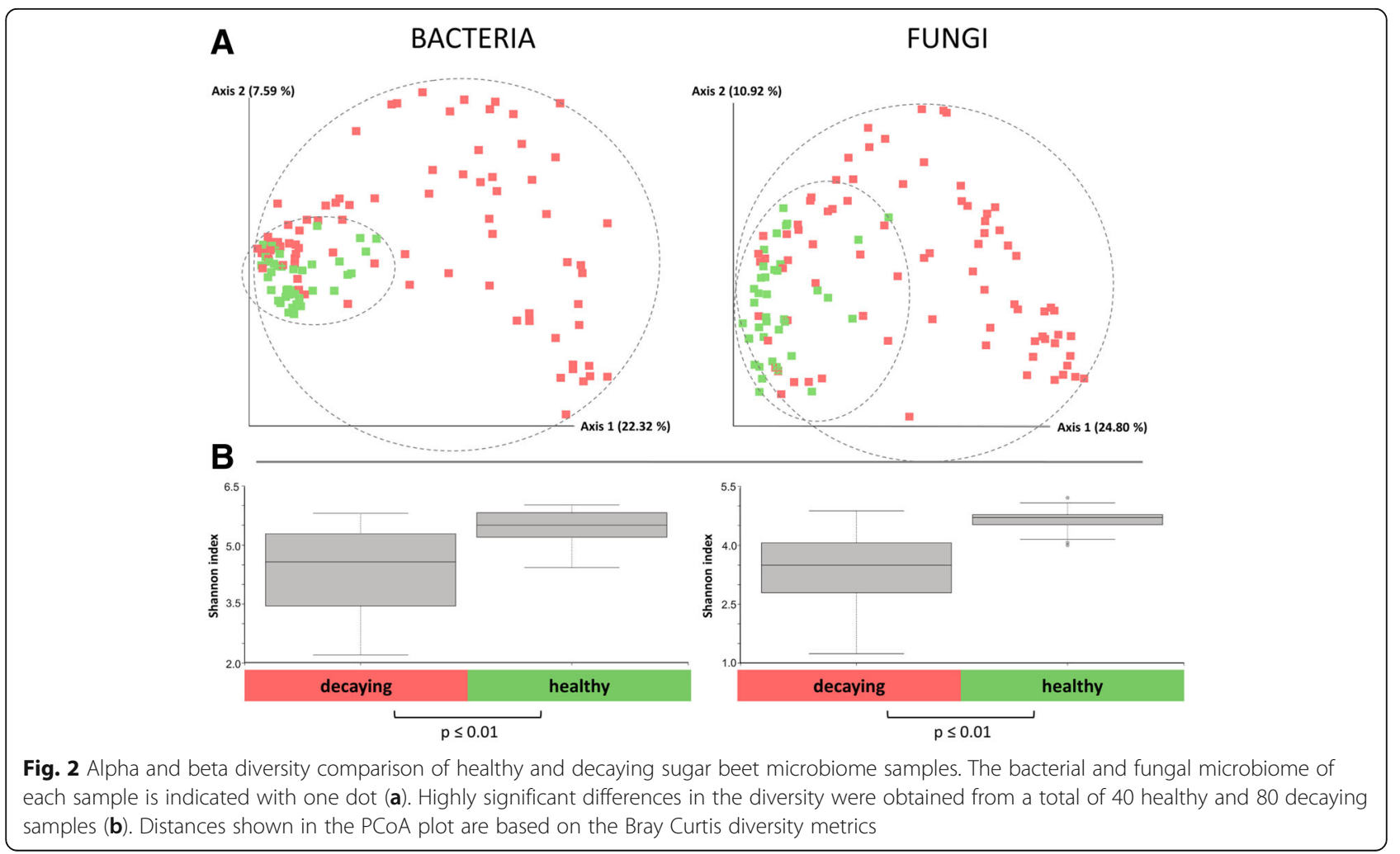


Sphingomonadales (4\%), and Rhizobiales (4\%). At order level, the most abundant taxa of healthy sugar beets were Flavobacteriales (21\%), Micrococcales (21\%), and Pseudomonadales (10\%), whereas the predominant taxa of decaying sugar beets were Lactobacillales (24\%), Rhodospirillales (20\%), and Flavobacteriales (9\%). At genus level Lactobacillus (18.4\%), Gluconobacter (16\%), and Leuconostoc (11.3\%) were the most abundant taxa in decaying samples, whereas Flavobacterium (20.6\%), Pseudarthrobacter (13.5\%), and Pseudomonas (9\%) were the most abundant taxa in healthy samples. (Fig. 3a).

The ITS dataset showed diversified fungal microbiomes in both healthy and decaying sugar beets. When the structure of the whole dataset was assessed, a total of $60-62 \%$ Ascomycota and 33\% Basidiomycota were observed within the fungal community. At class level, an increased fraction of Saccharomycetes $(+10 \%$ points; $12 \%$ total) and Eurotiomycetes (+ 9\% points; $10 \%$ total) as well as a decreased fraction of Sordariomycetes $(-16 \%$ points; $24 \%$ total) was found in the decaying samples. At order level, an increased abundance of Cystofilobasidiales $(+11 \%$ points; $21 \%$ total), Saccharomycetales $(+10 \%$ points; $12 \%$ total), and Eurotiales (+ $9.5 \%$ points; $10 \%$ total) was observed. At genus level, this resulted in an increased number of Candida (+ 7.5\%; 9.5 total), Penicillium (+ 9.5\%; 10\% total), Guehomyces (+5\%; 10\% total), and Mrakia (+5\%; $8 \%$ total). Healthy samples by contrast showed an increased amount of the genera Plectosphaerella $(+10 \% ; 21 \%$ total $)$ as well as Vishniacozyma $(+12 \%$; $18 \%$ in total). This was already shown in an increased abundance of the classes Sordariomycetes $(+16 \% ; 40 \%$ in total) as well as Tremellomycetes ( $+2 \%$; $30 \%$ in total). In comparison, at genus level, the most abundant genera in decaying samples were Plectosphaerella, Guehomyces, Candida, and Penicillium (all 10\%), whereas in healthy samples the genera Plectosphaerella (21\%) and Vishniacozyma (18\%) dominated (Fig. 3b).

\section{Trophic specialization in the fungal microbiome}

Taxonomic differences between healthy and decaying sugar beets were found to be accompanied by changes

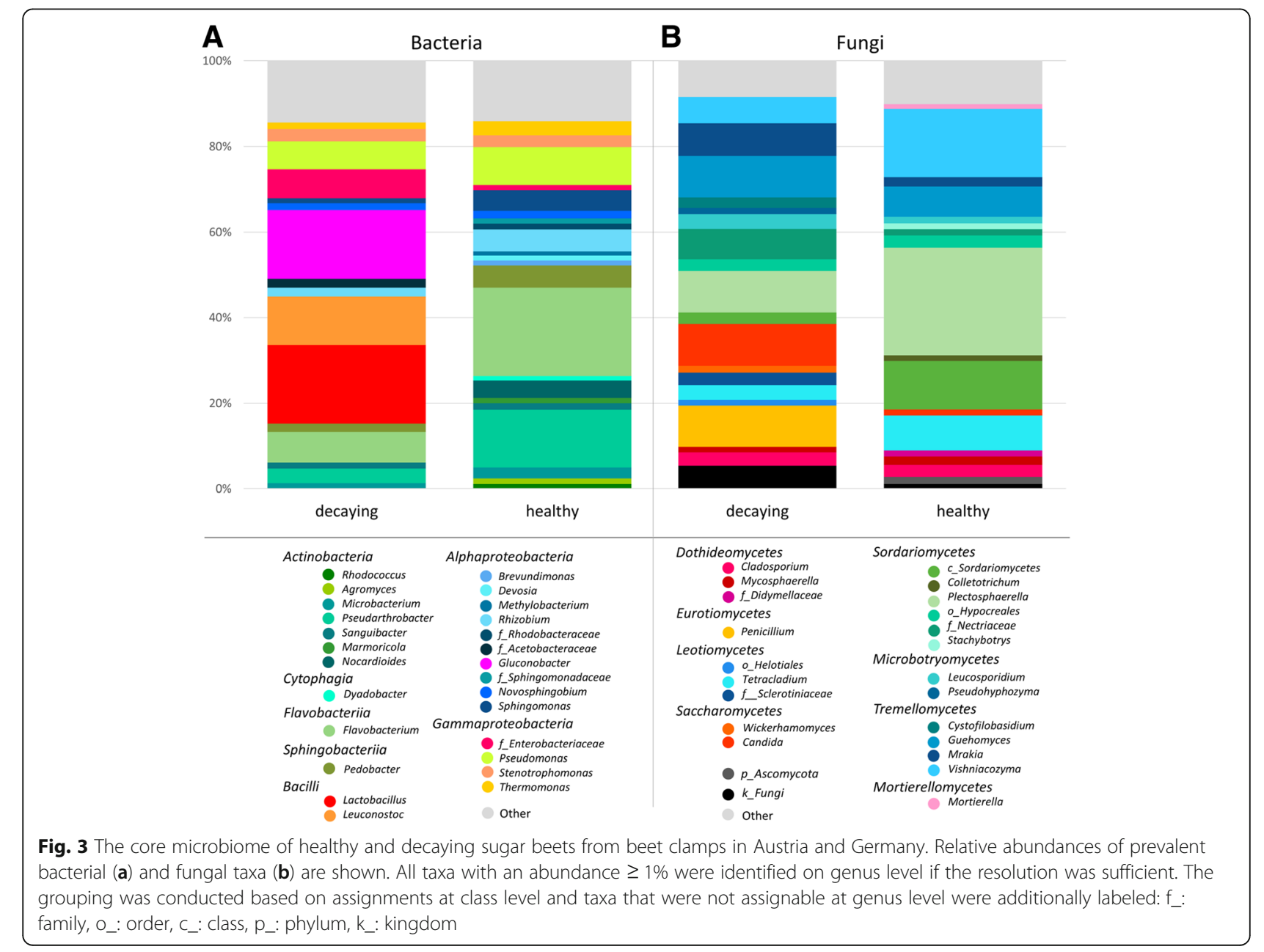


in the trophic modes of the identified core features. Healthy samples were mainly colonized by pathotrophic (24\%) and pathotrophic-saprotrophic-symbiotrophic (26\%) fungi. The trophic distribution in the decaying samples, however, was dominated by saprotrophic fungi (39\%) with a decreased fraction of pathotrophic (14\%) and pathotrophic-saprotrophic-symbiotrophic (12\%) fungi. Overall, a decrease in pathotrophic and symbiotrophic functions and an increase in saprotrophic functions from the microbiome in healthy to the microbiome in decaying sugar beets was observed (Fig. 4a).

\section{The health status of beets was identified as the major} driver for microbial community composition

The comparison of six different beet clamps in Austria and Germany showed significant differences in diversity as well as taxonomic composition. Health status explained the largest proportion of variance of the beets $(33.3 \%$ variation in 16 S dataset and $20.9 \%$ for ITS, $p \leq 0.001$ ). Different beet clamp sampling sites also explained 13.6\% variation in the $16 \mathrm{~S}$ and $21.7 \%$ variation in the ITS dataset $(p \leq 0.001)$, however, variances within the groups were higher $(F$ statistic $=3.43(16 \mathrm{~S})$ and 6.25 (ITS) compared to 56.36 (16S) and 30.91 (ITS) between health statuses). The country that sugar beet samples originated from accounted for the least variance $(5 \%$ in $16 \mathrm{~S}$ data and
$11.7 \%$ in ITS data, $p \leq 0.001$ ) (Fig. 4b, c; Additional file 1: Table S1). These findings were also reflected in $\beta$-diversity PCoA plots, where sample were separated by health status (Additional file 1: Figure S1, S2).

Samples obtained from the storage in Grossmugl (Austria) showed clear differences in the microbial composition when compared to the sampling spots located in lower Germany (Mittich, Kleinweichs, and Osterhofen). Sampling locations that were geographically located closer to each other (Additional file 1: Figure S3C), however, showed less significant differences. Overall, a change from relatively balanced abundances of bacterial taxa (microbiome of healthy sugar beets) to a predominance of Lactobacillales, as well as Rhodospirillales (decaying sugar beets) was evident for every sampling spot. The fungal community changed from a microbiome dominated by Vishniacozyma and Plectospaerella to an increasing number of Penicillium and Candida species (Fig. 5).

\section{Identification of disease indicators and correlation to sugar content in stored sugar beets}

Specific taxa, indicative either for the microbiome of healthy or decaying sugar beets, were selected based on the differences in their abundance in the representative samples (Figs. 3 and 5). Flavobacterium and Pseudarthrobacter within the bacterial community as well as Plectospaerella and Vishniacozyma within the fungal

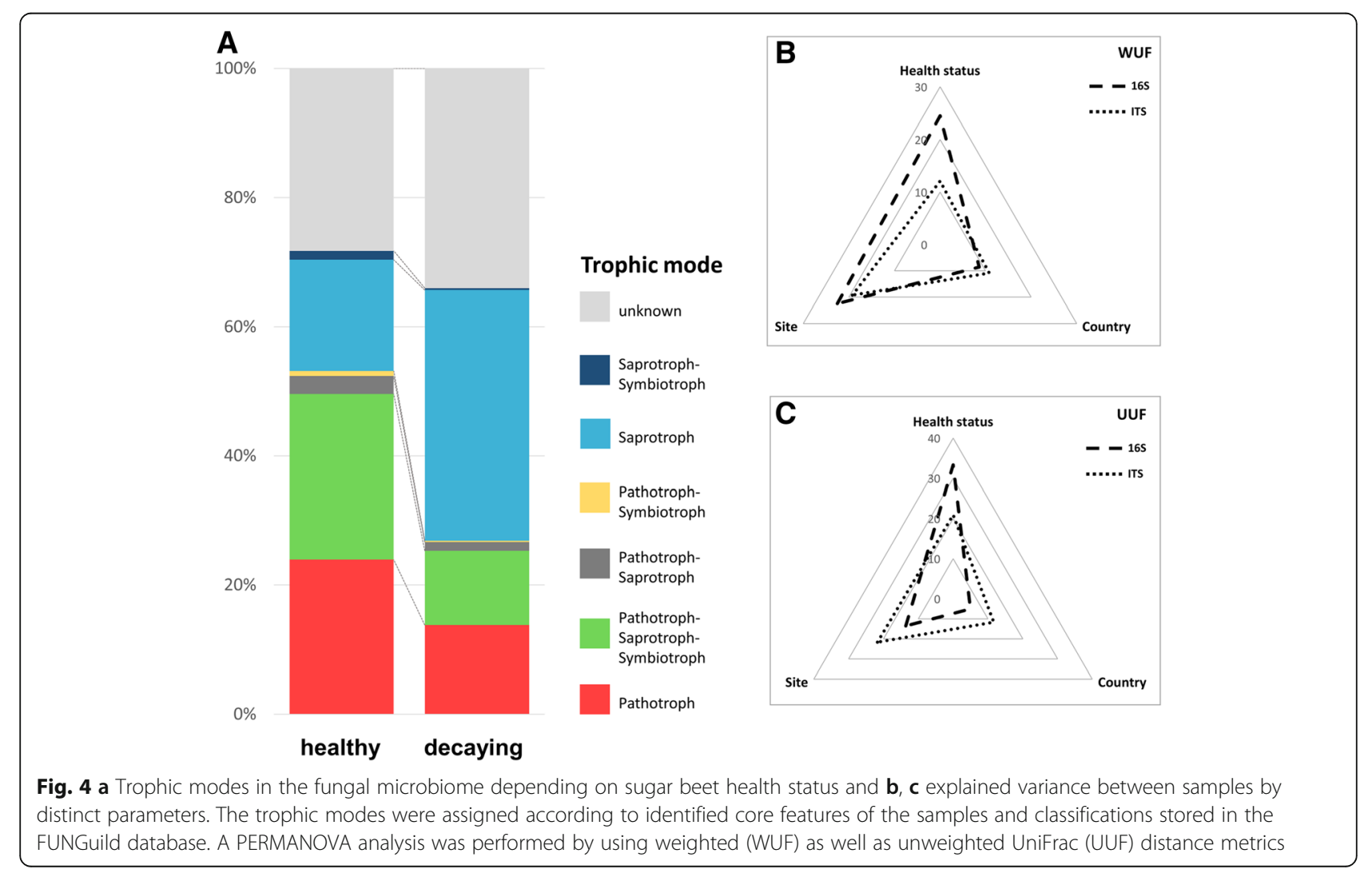



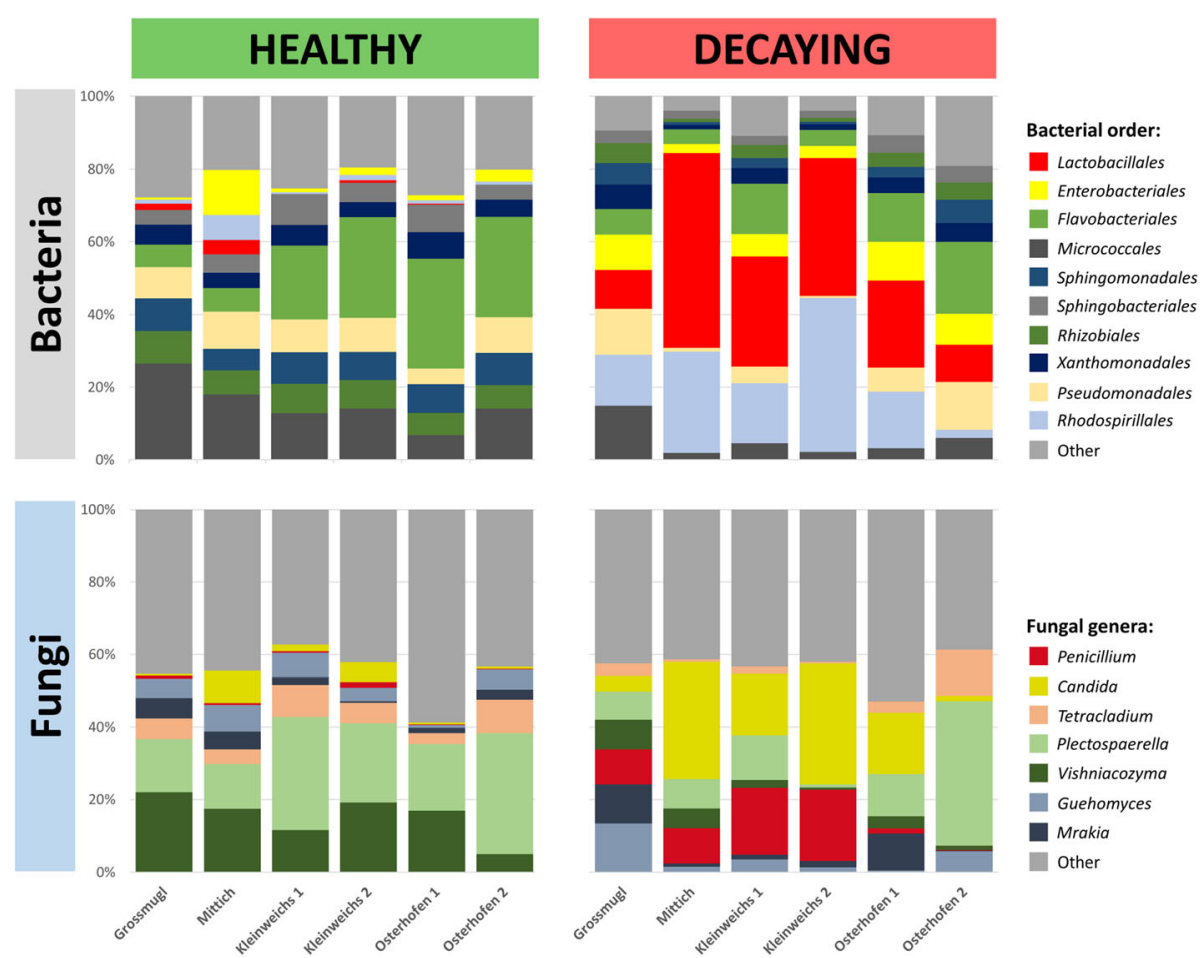

Fig. 5 Relative abundance of the most abundant ( $\geq 5 \%$ ) bacterial and fungal taxa in healthy and decaying sugar beet samples. Stored roots from six sugar beet clamps in Austria and Germany were analyzed by amplicon sequencing of the 16S rRNA gene fragment and the ITS region. The results were grouped according to the health status and the sampling site of the beets

community were found to be dominant in healthy sugar beets. In contrast, Lactobacillus and Gluconobacter as well as Candida and Penicillium were prevalent in decaying sugar beets. By implementing a real-time qPCR analysis with specific primers targeting microbial indicators in stored sugar beets, the gradual increase of disease indicators and simultaneous loss of health indicators was shown. During a 3-month storage trial, an increase of Candida $\left(10^{5}\right.$ to $5 \times 10^{6}$ copies/g), Fusarium $\left(2 \times 10^{3}\right.$ to $10^{4}$ copies/g), and Penicillium (0 to $10^{4}$ copies/g) and simultaneous decrease of Vishniacozyma $\left(10^{5}\right.$ to $5 \times 10^{4}$ copies/g) was observed (Fig. 6a). In case of Plectosphaerella, an initial decreases in abundance $\left(2 \times 10^{5}\right.$ to $10^{5}$ copies/g), but overall constant abundances $\left(10^{5}\right.$ copies $\left./ g\right)$ throughout the storage period were found.

In order to verify the disease progress in the samples that were used for qPCR primer evaluations, complementary analytical analyses of beet carbohydrates were conducted with stored samples. The sugar content of sugar beets stored under controlled conditions showed a decreasing concentration of sucrose (-3\% points) during the storage period of 3 months. At the same time, an increase of inverted sugars (glucose (2 to $14 \mathrm{~g} / \mathrm{kg}$ ) and fructose (1.5 to $14 \mathrm{~g} / \mathrm{kg})$ ) was observed (Fig. 6b).

\section{Discussion}

Fungal pathogens prevail among isolates from decaying sugar beets

The obtained results of the present study provide the first detailed microbiome characterization of conventionally stored sugar beets in an industrially scaled, uncontrolled environment. By combining different methods, a holistic assessment of the fungal microbiome in decay-affected sugar beets was constructed. $18 \mathrm{~S}$ gene sequencing data of 120 fungal isolates was compared to ITS next-generation amplicon data. In comparison, $86 \%$ of isolated fungi identified on genus level were also found in the amplicon libraries. The cultivation-dependent identification of fungal isolates showed a prevalence of certain taxa such as Penicillium and Fusarium, when compared to the amplicon sequencing dataset. This likely resulted from the specific procedure during the isolation process that could have affected the frequency of isolated strains. While only homogenized peel was used for the total DNA extraction, also surface-sterilized fragments of infected sugar beets were placed on agar plates during isolation. This could have facilitated the isolation of Fusarium species, since this pathogen primarily colonizes the plant endosphere [23]. In the case of Penicillium, its high spore production allows it to 


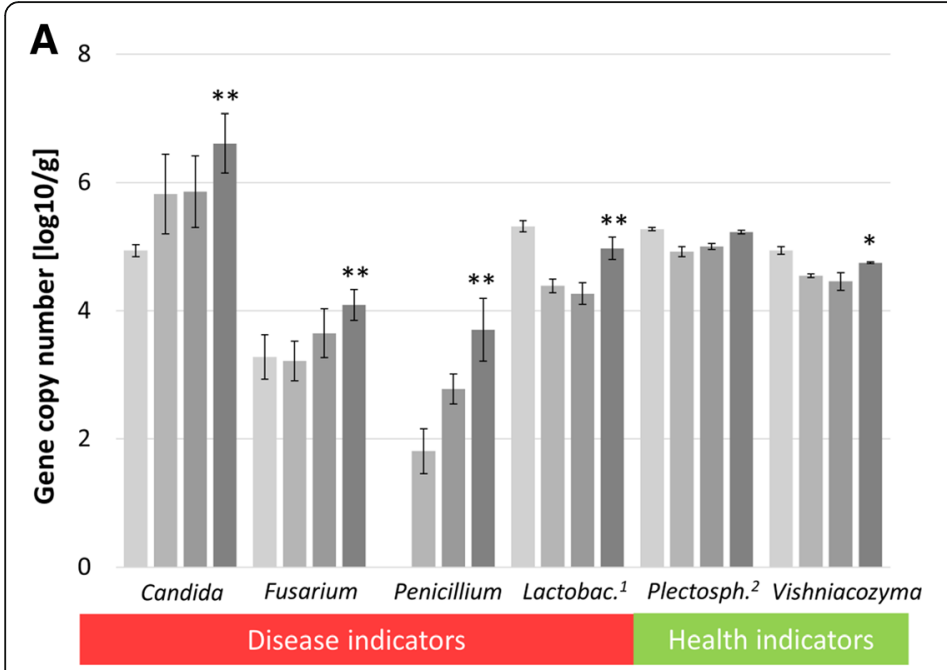

B

20

${ }^{1}$ Lactobac, $=$ Lactobacillus; ${ }^{2}$ Plectosph. $=$ Plectosphaerella
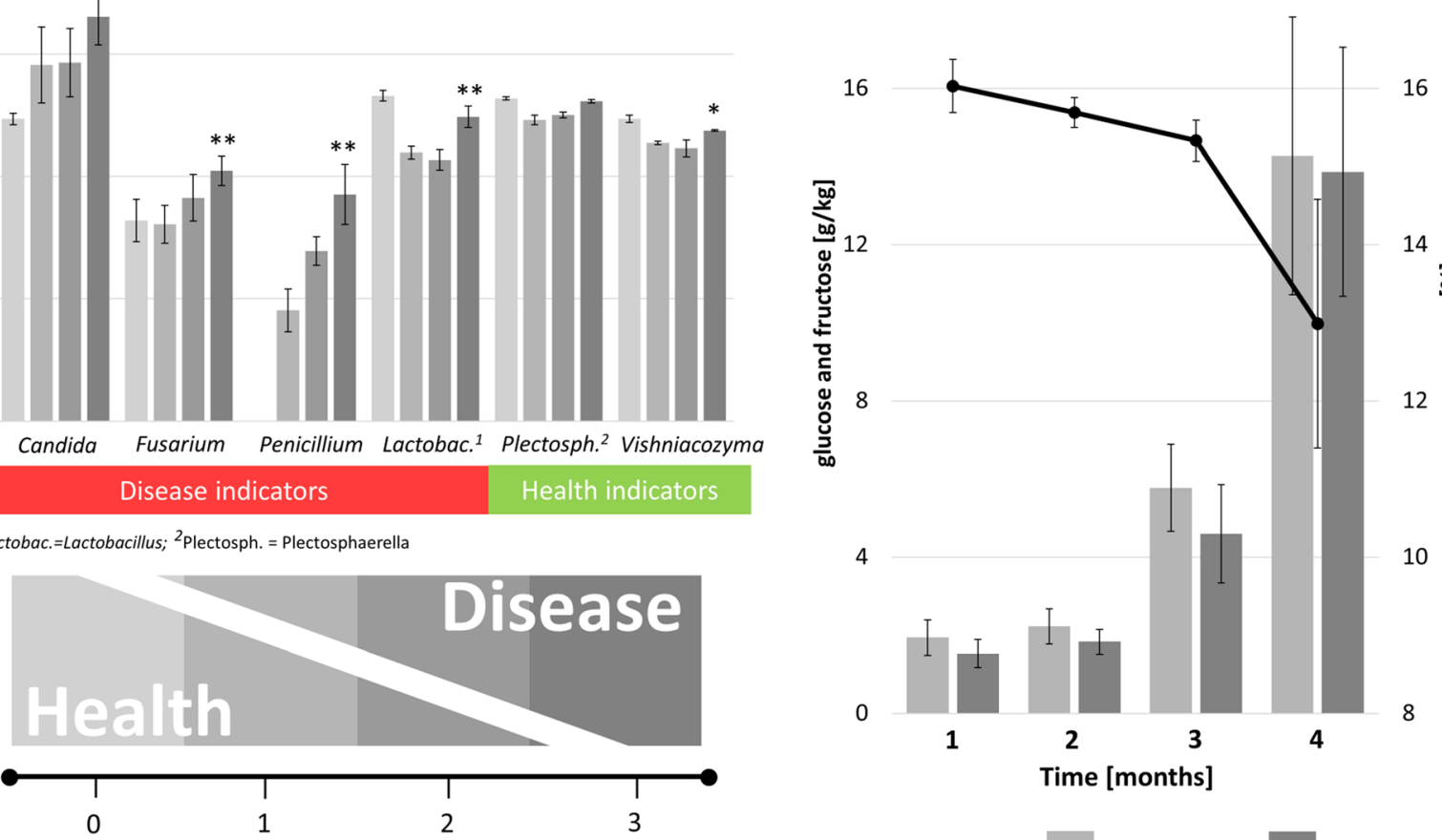

Time [months]

- Sucrose

Glucose

Fructose

Fig. 6 Real-time gPCR analysis of bacterial and fungal indicator taxa in stored sugar beets (a) and analyzed sucrose, glucose, and fructose contents in sugar beets (b). Gene copy numbers per gram sugar beet surface showed distinct tendencies related to accumulations of health and disease indicators during three months of controlled storage (color gradient). Statistical significance between the first and last measurement was tested using the Student's $t$ test: $p$ value $<0.01{ }^{*} ; p<0.05^{*}$

overgrow slow-growing fungal taxa and likely lead to its isolation in higher proportions. The fungal ITS library obtained with high-throughput sequencing showed overall a higher diversity of different fungal taxa, which is partially due to cultivability limitations of certain taxa on standard isolation media $[24,25]$.

\section{Bacterial diversity decrease was accompanied by an emergence of several highly abundant lineages}

Microbial diversity as well as distinct changes in the microbial community were previously shown to be linked to disease incidence $[5,26]$. The data obtained in this study supports the hypothesis that lower diversity in the bacterial as well as fungal community is connected to a higher sensitivity to microbiome shifts that substantially alter the community structure. The lower diversity in decaying samples was reflected by a significant decrease in diversity indices in both the bacterial and the fungal dataset. In analogy to our findings, changed microbial diversity was found in stored onions when comparing healthy and diseased ones and fungal diversity was found to be higher in roots of healthy winter wheat plants [27, 28]. Moreover, a decrease in diversity was shown to facilitate invasion of pathogenic species into communities [29].

Even though amplicon-based sequencing can be affected by certain biases [30], the taxonomic composition of the bacterial as well as fungal beet microbiome, obtained with this dataset, was primarily linked to the health status of the sampled sugar beets. The geographic location of the beet clamps played a less significant role for the observed variability. Similarly, also Yurgel and colleagues (2018) observed taxonomic changes based on health status in stored onions [28]. Additionally, Liebe et al. (2016) already observed a similar effect in sugar beets when stored at different temperatures [14]. Depending on the storage conditions, the analyzed beets harbored specific fungal taxa, whereas the originating environment was less influential. In this study, sugar beets, stored under representative conditions without any protection from adverse environmental factors (moisture, temperature fluctuations, frost, etc.), showed a fungal community dominated by Candida, Penicillium, Guehomyces, and Plectosphaerella sp. in decaying sugar beets. The fungal microbiome of sampled healthy beet roots was, interestingly, comparable with the analyzed reference sugar beets 
in Liebe et al. (2016) harnessing mostly Plectosphaerella sp. [14]. The observed taxonomic changes were also reflected by trophic modes within the fungal community. Dominant pathotrophic and pathotrophic-saprotrophicsymbiotrophic functions in healthy samples were replaced by saprotrophic functions in decaying sugar beets. Similar findings were also made by $\mathrm{Yu}$ and colleagues (2012) linking the prevalence of saprotrophic fungi mostly to diseased pea plants, the abundance of pathogenic fungi, however, not to a specific health status [26].

\section{Identification of health indicators in the microbiome of sugar beets}

Different potential biological markers were identified by contrasting healthy and diseased samples of stored sugar beets. Distinct taxa were shown to be highly abundant in samples representing each disease condition. The necrotrophic fungal lineage Plectosphaerella, found in healthy beets, was previously shown to be a growth-promoting microbe in sugar beets [31]. Moreover, it was reported as a potential biological control agent against potato cyst nematodes as well as a potential bioherbicide [32,33]. Previous studies on sugar beet storage observed this taxon mostly in sugar beets before storage [14]. Other health-related taxa, such as Flavobacterium and Pseudarthrobacter, were often reported in the rhizosphere of different plants as well as their involvement in plant defense mechanisms or growth promotion [34-37]. Other taxa, associated with decaying sugar beets, such as Penicillium, are typical saprophytic fungi and postharvest pathogens and were observed previously in rotting sugar beet after harvest [14, 38, 39]. Lactobacillus as well as the fungal genus Candida were predominantly detected in decaying sugar beets and are associated with sugar fermentation to acid or alcohol compounds and are unwanted in stored sugar beets because of this activity $[40,41]$. We hypothesize that such taxa occur on decaying sugar beets primarily due to increased free monosaccharides originating from the hydrolyzation processes of sucrose by fungal extracellular proteins.

Real-time qPCR analyses conducted on the basis of the identified health and disease indicators in stored sugar beets provided a first evidence for the applicability of such indicators for agricultural management strategies. The data was obtained within small-scale experiments and must be further expanded in upcoming approaches to confirm the reliability of the indicators for industry-scale applications. During the representative storage period of three months, health-related indicators were either decreasing or remained constant. In contrast, disease-related indicators increased substantially over the storage period. The quantitative analysis of these taxa indicated a gradual disease development that is linked to microbial sucrose concentration loss and simultaneously increase in inverted sugars during storage [42], which was confirmed by targeted analyses in the present study.

\section{Conclusion}

Storage rot in stored sugar beets was shown to be accompanied by a change in microbial abundances. The present study highlighted substantial shifts within the bacterial as well as fungal community that correlated to decay incidence in stored roots. Changes in the prevalence of certain taxa can potentially indicate decay development at an early stage and facilitate an implementation of targeted countermeasures. Taxonomic changes were shown to be accompanied by trophic specialization in the fungal community. For upcoming postharvest applications, the novel insights provide a basis to design suitable biocontrol agents maintaining the balance of taxa associated with the microbiome of healthy sugar beets and preventing the establishment of degrading microorganisms. Furthermore, the identification of diseases indicators can be used as decision tool and supports the prioritization of processing of harvested beets during storage management. Additional studies are needed to confirm the implementability of the obtained results and to assign levels of quantitative measurements, which will allow to indicate the degree of disease.

\section{Methods}

\section{Sampling of sugar beets and isolation of fungi}

Healthy $(n=40)$ and decaying $(n=80)$ sugar beets were obtained from beet clamps in Austria (Upper Austria) and Germany (Bavaria). The detailed sampling locations are provided in Additional file 1: Table S2 and Figure S3C. Decaying sugar beets were obtained from nests of fungal mycelia in the beet clamps (Additional file 1 : Figure S3A, B). Samples with severe and intermediate fungal infection were selected. Healthy sugar beets were collected from the non-infected, symptoms-free surrounding area of infected beet clamps. Following the sampling, $20 \mathrm{~g}$ of the sugar beet skin (surface of tap root and stem end) was peeled and washed with $50 \mathrm{~mL}$ of $0.85 \%$ sodium chloride solution in a stomacher (BagMixer; St. Nom, France) for $3 \mathrm{~min}$. The obtained solution was prepared for total community DNA extraction as described later. A total of $100 \mu \mathrm{L}$ of the solution obtained from decaying sugar beets was plated on SNA plates [43] containing penicillin $\mathrm{G}(100 \mu \mathrm{g} / \mathrm{mL})$, dihydrostreptomycinsulfate $(50 \mu \mathrm{g} / \mathrm{mL})$, and chlortetracycline $(10 \mu \mathrm{g} / \mathrm{mL})$ in serial $1 / 10$ dilutions until a final dilution of $10^{-10}$ was reached. In addition, surface sterilized (submerged in $4 \%$ sodium hypochlorite, $5 \mathrm{~min}$ ) and washed (two times sterile distilled water) beet sections from diseased beets were placed on a SNA plate to obtain fungal isolates growing in the beet endosphere. A total of ten fungal strains per sugar 
beet were randomly picked based on morphology from the plates and further subcultured on PDA, SNA, and water agar plates (tap water $+18 \mathrm{~g} / \mathrm{L}$ agar). The strains were further grouped using morphologic clustering after inspecting the single isolates on the different plates. Several strains of each morphologic cluster (120 strains in total) were subjected to $18 \mathrm{~S}$ rRNA gene fragment Sanger sequencing (LGC Genomics, Berlin, Germany). Quality checked sequences were blasted against the NCBI database as well as the UNITE v7 database [44].

\section{Storage of sugar beets under controlled conditions}

A total of 20 untreated and undamaged sugar beets harvested from a single field in Germany (Rhenish Hesse, Rhineland-Palatinate; $49^{\circ} 35^{\prime} 54.388^{\prime \prime} \mathrm{N}, 8^{\circ} 12^{\prime}$ $48.823^{\prime \prime}$ E) were stored directly after harvest under controlled condition at $8{ }^{\circ} \mathrm{C}$ and $75 \%$ relative humidity for 3 months. Sampling of five sugar beets at the beginning (T0) and every 30 days (T1, T2, and T3) was performed as described above. A total of $20 \mathrm{~g}$ of sugar beet peel was washed in a stomacher with $50 \mathrm{~mL}$ of sodium chloride $(0.85 \%)$. A total of $4 \mathrm{~mL}$ of the solution was centrifuged into a pellet and further used for community DNA extraction. Sugar content in the sugar beet flesh was measured using standardized ICUMSA (International Commission for Uniform Methods of Sugar Analysis) methods for the determination of glucose and fructose by enzymatic assays and the polarization of sugar (sucrose) by the cold aqueous digestion method $[45,46]$.

\section{Total community DNA extraction and construction of amplicon library}

A total of $4 \mathrm{~mL}$ of the obtained washing solution from the sampling step was centrifuged $\left(13,000 \times g, 20 \mathrm{~min}, 4{ }^{\circ} \mathrm{C}\right)$ and the pellet was stored at $-70{ }^{\circ} \mathrm{C}$ until further use. Using the FastDNA ${ }^{\circ}$ Kit for Soil (MP Biomedicals/USA) genomic DNA was extracted from all samples. All steps were conducted as stated in the manufacturer's protocol. Following DNA extraction, the $16 \mathrm{~S}$ rRNA primers $514 \mathrm{f}$ and 926r (GTGYCAGCMGCCGCGGTAA; CCGYCAAT TYMTTTRAGTTT) and the ITS primer pair ITS1f and ITS2r (CTTGGTCATTTAGAGGAAGTAA; GCTGCG TTCTTCATCGATGC) were used in PCR for amplicon library construction. As described in the protocols and standards section of the Earth microbiome project [47], both primer pairs were modified with specific primer pads (TATGGTAATT/AGTCAGCCAG) and linker (GT/GG) for the attachment of a Golay barcode sequences. Two consecutive PCR reactions were performed and all PCR reactions, conducted in triplicates were pooled after the second PCR. The first PCR (amplification of the V4 and V5 region or ITS1 region) was performed in a total volume of $10 \mu \mathrm{L}(1 \mu \mathrm{L}$ DNA, $2 \mu \mathrm{L}$ Taq\&Go, $0.1 \mu \mathrm{L}$ of each Primer, $0.15 \mu \mathrm{L}$ of mPNA and pPNA, and $6.5 \mu \mathrm{L}$ of water).
Added blocking primers mPNA and pPNA prevented the amplification of mitochondrial and chloroplast DNA [48]. The reactions were performed on a Whatman Biometra ${ }^{\circ}$ Tpersonal and Tgradient thermocycler (Biometra $\mathrm{GmbH}$, Göttingen, Germany) with the following settings: $95^{\circ} \mathrm{C}$ for $45 \mathrm{~s}, 78{ }^{\circ} \mathrm{C} 5 \mathrm{~s}, 55^{\circ} \mathrm{C} 45 \mathrm{~s}, 72{ }^{\circ} \mathrm{C} 90 \mathrm{~s}(35 \times)$, including an initial denaturation of $5 \mathrm{~min}$ at $95^{\circ} \mathrm{C}$ and a final extension of $5 \mathrm{~min}$ at $72{ }^{\circ} \mathrm{C}$. A second PCR step (multiplexing with Golay barcodes) a total volume of $30 \mu \mathrm{L}$ ( $2 \mu \mathrm{L}$ of the first PCR (template), $6 \mu \mathrm{L}$ Taq\&Go, $1.2 \mu \mathrm{L}$ of barcode-primers and $19.6 \mu \mathrm{L}$ of water) run at the following settings: $95^{\circ} \mathrm{C}$ for $30 \mathrm{~s}, 53{ }^{\circ} \mathrm{C} 30 \mathrm{~s}, 72{ }^{\circ} \mathrm{C} 30 \mathrm{~s}(15 \times)$, including an initial denaturation of $5 \mathrm{~min}$ at $95{ }^{\circ} \mathrm{C}$ and a final extension of 5 min at $72{ }^{\circ} \mathrm{C}$. After each PCR amplification step, the quality was checked by gel electrophoresis. All tree replicates of quality checked PCRs from each sample were pooled and purified using the Wizard SV Gel and PCR Clean-Up System (Promega, Madison, USA) according to the protocol. Equimolar DNA concentrations of each barcoded amplicon sample were sent to GATC Biotech AG, Konstanz, Germany. After entry quality control and adapter ligation, 16S rRNA and ITS gene amplicons were sequenced on an Illumina HiSeq instrument.

\section{Data evaluation using bioinformatics tools}

Data obtained with Illumina HiSeq amplicon sequencing was analyzed with QIIME 2 (2018.6 release) and QIIME 1.9.1 [49] according to tutorials provided by the QIIME developers. After joining forward and reversed reads and barcode extraction in QIIME 1.9.1, the data was imported into QIIME 2 for further analysis. After demultiplexing, the DADA2 algorithm [50] was applied to denoise and truncate the reads and summarize sequence variants (SVs) in a feature table. To increase the quality, chimeric data was filtered as well as mitochondria and chloroplast reads (for $16 \mathrm{~S}$ data) or bacteria and archaea reads (for ITS data) were discarded. A total of 3489 ITS and 8935 16S SVs were assigned for a total of 16,155,698 ITS and 4,036,955 16S reads (Additional file 1: Table S3). Alpha diversity, beta diversity, as well as statistical analysis was performed using the QIIME2 core diversity metrics. Naïve-Bayes classifier were trained on the SILVA v128 [51] at 99\% similarity as well as the UNITE v7.2 [44] database for taxonomic assignment. Subsequently, core microbiomes (features present in at least $50 \%$ of the samples) were calculated for each group (healthy and decaying) and exported for display in bar charts. Functional analysis of fungal feature tables was performed using the FUNGuild online tool [52].

\section{Statistical analysis of bioinformatics data}

Alpha and beta diversity was tested in QIIME 2. Therefore, the Kruskal-Wallis (alpha) and the anosim test 
Table 1 Sequences, annealing temperatures, fragment length, and sources of the implemented qPCR primers. The primers for Vishniacozyma and Plectospaerella were designed with deposited sequences (accession numbers provided) in the NCBI database and the Primer-BLAST tool [53]

\begin{tabular}{|c|c|c|c|c|c|}
\hline $\begin{array}{l}\text { Taxonomic } \\
\text { group }\end{array}$ & Forward primer & Reverse primer & $\begin{array}{l}\text { Length } \\
\text { (bp) }\end{array}$ & $\begin{array}{l}\text { Annealing temp } \\
\left({ }^{\circ} \mathrm{C}\right)\end{array}$ & Reference/source \\
\hline Vishniacozyma & CGCATCGATGAAGAACGCAG & AAAACCCAAGTGGGGTGAGG & 151 & 64.6 & $\begin{array}{l}\text { NR_073260.1, this } \\
\text { study }\end{array}$ \\
\hline Plectospaerella & ATCTCTTGGCTCCAGCATCG & GATACTGGAAGGCGCCATGT & 112 & 65 & GU724980.1, this study \\
\hline Candida & TCTAACGTCTATGCGAGTG & ATACCCAAATTCGACGATCG & 244 & 59.4 & [54] \\
\hline Fusarium & CAACTCCCAAACCCCTGTGA & GCGACGATTACCAGTAACGA & 398 & 58 & {$[55]$} \\
\hline Lactobacillus & GCAGCAGTAGGGAATCTTCCA & GCATTYCACCGCTACACATG & 342 & 62.1 & {$[56]$} \\
\hline Penicillium & $\begin{array}{l}\text { ATGAAATCCTCCCTGTGGGTTA } \\
\text { GT }\end{array}$ & $\begin{array}{l}\text { GAAGGATAATTCCGGGGTAGTCA } \\
\pi\end{array}$ & 92 & 65 & [57] \\
\hline
\end{tabular}

(beta) were used. Variance explained by parameters was analyzed with a PERMANOVA test in QIIME. Significant taxonomic differences between the groups were observed with the ANCOM test in QIIME 2.

\section{Real-time qPCR measurement targeting microbial indicators}

Following the community DNA extraction from stored sugar beet samples obtained under controlled conditions, qPCR amplifications using specific primers were conducted in order to quantify distinct taxonomic groups that were selected as disease indicators. Specific primers targeting Candida, Fusarium, Penicillium, Lactobacillus, as found in previous literature were implemented. Primers for Vishniacozyma and Plectosphaerella were designed using the Primer-BLAST tool [53] and deposited sequences in the NCBI database (Table 1). The quantification was performed with a Corbett Research TM thermocycler (Rotor-Gene 6000, Corbett Research, UK) and SYBR Green PCR master mix TM (KAPA Biosystems, USA). The standard curves were obtained using a single isolate gene fragment with known copy numbers and further 1:10 dilutions. Three replicates of each standard dilution were prepared to calculate mean values. The standards were employed to determine the gene copy numbers in the analyzed samples. Negative controls (using pure $\mathrm{dH}_{2} \mathrm{O}$ ) were implemented and further subtracted from the analyzed samples to reduce quantification inaccuracies.

\section{Additional file}

Additional file 1: Table S1. Summary of performed PERMANOVA test. Pairwise comparison of categories using the unweighted (UUF) and weighted (WUF) UniFrac distance metrics for both, the 165 and ITS, datasets. Table S2: Sampling locations and sample conditions of the implemented sugar beets. Healthy and decaying beets were sampled from beet clamps in Austria (AT) and Germany (DE). At the locations Kleinweichs and Osterhofen, two neighboring beet clamps were sampled (1 and 2). Table S3: Overview of sequencing data. Number of reads, assigned sequence variants (SVs) using the DADA2 algorithm and Shannon Index of each group is given. Figure S1: Principal component analysis of bacterial and fungal communities from different beet clamps. PCoA using the unweighted UniFrac (UUF) distance metric. Samples are color-coded based on their geographic origin or health status. Figure S2: Principal component analysis of bacterial and fungal communities from different beet clamps. PCoA using the weighted UniFrac (WUF) distance metric. Samples are color-coded based on their geographic origin or health status. Figure S3: Sample visualization, schematic representation of fungal growth in the beet clamps, and geographic locations of the sampling sites. Fungal nests start within the clamp and spread to the surrounding beets (A, B). Healthy, uninfected beets, as well as decaying sugar beets within the same beet clamp were sampled from six different beet clamps in Austria and Germany (C). (DOCX 1605 kb)

\section{Abbreviations}

DNA: Deoxyribonucleic acid; ITS: Internal transcribed spacer; PCR: Polymerase chain reaction; qPCR: Real-time (quantitative) polymerase chain reaction

\section{Acknowledgements}

The authors gratefully acknowledge Anna Heinzel (Graz) and Barbara Fetz (Graz) for the help with laboratory work, Sebastian Siebauer (Plattling) for his help during sampling, as well as Stefan Hofmann (Offstein) for the help with sugar analysis.

\section{Funding}

This work has been supported by the Federal Ministry of Science, Research and Economy (BMWFW), the Federal Ministry of Traffic, Innovation and Technology (bmvit), the Styrian Business Promotion Agency SFG, the Standortagentur Tirol, the Government of Lower Austria, and ZIT

- Technology Agency of the City of Vienna through the COMET-Funding Program managed by the Austrian Research Promotion Agency FFG (grant number 282482).

\section{Availability of data and materials}

Raw sequencing data for each sample used in this study was deposited at the European Nucleotide Archive (ENA) in the FASTA format and is available under the Bioproject accession number PRJEB28964.

\section{Authors' contributions}

$\mathrm{CZ}, \mathrm{GB}$, and TC conceived and designed the study. $\mathrm{KH}$, JM, and HE provided specific knowledge for improved study design and oversaw the long-term storage experiments. PK performed the laboratory work as well as evaluation of the microbiome data. PK, TC, CZ, and GB wrote the manuscript. All authors read and approved the final version of the manuscript.

Ethics approval and consent to participate Not applicable. 


\section{Consent for publication}

Not applicable.

\section{Competing interests}

The authors declare that they have no competing interests.

\section{Publisher's Note}

Springer Nature remains neutral with regard to jurisdictional claims in published maps and institutional affiliations.

\section{Author details}

${ }^{1}$ Austrian Centre of Industrial Biotechnology, Petersgasse 14, 8010 Graz, Austria. ${ }^{2}$ Institute of Environmental Biotechnology, Graz University of Technology, Petersgasse 12, 8010 Graz, Austria. ${ }^{3}$ Südzucker AG, Maximilianstraße 10, 68165 Mannheim, Germany. ${ }^{4}$ Agrana Research \& Innovation Center, Josef-Reither-Straße 21 - 23, 3430 Tulln, Austria. ${ }^{5}$ Roombiotic GmbH, c/o: SciencePark, Stremayrgasse 16/IV, 8010 Graz, Austria.

Received: 16 April 2019 Accepted: 31 July 2019

\section{Published online: 07 August 2019}

\section{References}

1. Berendsen RL, Pieterse CMJ, Bakker PAHM. The rhizosphere microbiome and plant health. Trends Plant Sci. 2012;17:478-86.

2. Berg G, Grube M, Schloter M, Smalla K. Unraveling the plant microbiome: looking back and future perspectives. Front Microbiol. 2014;5 https://doi. org/10.3389/fmicb.2014.00148.

3. Vandenkoornhuyse P, Quaiser A, Duhamel M, Le Van A, Dufresne A. The importance of the microbiome of the plant holobiont. New Phytol. 2015; 206:1196-206.

4. Droby S, Wisniewski M, Teixidó N, Spadaro D, Jijakli MH. The science, development, and commercialization of postharvest biocontrol products. Postharvest Biol Technol. 2016;122:22-9.

5. Berg G, Köberl M, Rybakova D, Müller H, Grosch R, Smalla K. Plant microbial diversity is suggested as the key to future biocontrol and health trends. FEMS Microbiol Ecol. 2017:93 https://doi.org/10.1093/femsec/fix050.

6. Castoria R, De Curtis F, Lima G, Caputo L, Pacifico S, De Cicco V. Aureobasidium pullulans (LS-30) an antagonist of postharvest pathogens of fruits: study on its modes of action. Postharvest Biol Technol. 2001;22:7-17.

7. Tzortzakis NG, Economakis CD. Antifungal activity of lemongrass (Cympopogon citratus L.) essential oil against key postharvest pathogens. Innov Food Sci Emerg Technol. 2007;8:253-8.

8. Trebbi D, McGrath JM. Fluorometric sucrose evaluation for sugar beet. J Agric Food Chem. 2004;52:6862-7.

9. Osburn RM, Schroth MN, Hancock JG, Hendson M. Dynamics of sugar bee seed colonization by Pythium ultimum and Pseudomonas species: effects on seed rot and damping-off. Phytopathology. 1989:79:709-16.

10. Kiewnick S, Jacobsen BJ, Braun-Kiewnick A, Eckhoff JLA, Bergman JW. Integrated control of Rhizoctonia crown and root rot of sugar beet with fungicides and antagonistic bacteria. Plant Dis. 2001;85:718-22.

11. Weiland J, Koch G. Sugarbeet leaf spot disease (Cercospora beticola Sacc.)t. Mol Plant Pathol. 2004:5:157-66.

12. Zachow C, Fatehi J, Cardinale M, Tilcher R, Berg G. Strain-specific colonization pattern of Rhizoctonia antagonists in the root system of sugar beet. FEMS Microbiol Ecol. 2010;74:124-35

13. Jaggard KW, Clark CJA, May MJ, McCullagh S, Draycott AP. Changes in the weight and quality of sugarbeet (Beta vulgaris) roots in storage clamps on farms. J Agric Sci. 1997;129:287-301.

14. Liebe S, Wibberg D, Winkler A, Pühler A, Schlüter A, Varrelmann M. Taxonomic analysis of the microbial community in stored sugar beets using high-throughput sequencing of different marker genes. FEMS Microbiol Ecol. 2016;92 https://doi.org/10.1093/femsec/fiw004.

15. Klotz KL, Finger FL. Impact of temperature, length of storage and postharvest disease on sucrose catabolism in sugarbeet. Postharvest Biol Technol. 2004:34:1-9.

16. Hoffmann C. Lagerfähigkeit geköpfter und entblätterter Rüben. Sugar Ind. 2012:137:458-67.

17. Kenter C, Hoffmann CM. Changes in the processing quality of sugar beet (Beta vulgaris L.) during long-term storage under controlled conditions. Int J Food Sci Technol. 2009;44:910-7.
18. Zachow C, Tilcher R, Berg G. Sugar beet-associated bacterial and fungal communities show a high indigenous antagonistic potential against plant pathogens. Microb Ecol. 2008;55:119-29.

19. Berg G. Plant-microbe interactions promoting plant growth and health: perspectives for controlled use of microorganisms in agriculture. Appl Microbiol Biotechnol. 2009:84:11-8.

20. Janisiewicz WJ, Korsten L. Biological control of postharvest diseases of fruits. Annu Rev Phytopathol. 2002;40:411-41.

21. Kusstatscher P, Cernava T, Harms K, Maier J, Eigner H, Berg G, et al. Disease incidence in sugar beet fields is correlated with microbial diversity and distinct biological markers. Phytobiomes J. 2019; https://doi.org/10.1094/ PBIOMES-01-19-0008-R

22. Schillinger $U$, Geisen $R$, Holzapfel $W H$. Potential of antagonistic microorganisms and bacteriocins for the biological preservation of foods. Trends Food Sci Technol. 1996;7:158-64.

23. Zhang X-W, Jia L-J, Zhang Y, Jiang G, Li X, Zhang D, et al. In planta stage -specific fungal gene profiling elucidates the molecular strategies of Fusarium graminearum growing inside wheat coleoptiles. Plant Cell. 2012; https://doi.org/10.1105/tpc.112.105957.

24. Müller T, Ruppel S. Progress in cultivation-independent phyllosphere microbiology. FEMS Microbiol Ecol. 2014:87:2-17.

25. Wu P-C, Su H-JJ, Ho H-M. A comparison of sampling media for environmental viable fungi collected in a hospital environment. Environ Res. 2000;82:253-7.

26. Yu L, Nicolaisen M, Larsen J, Ravnskov S. Molecular characterization of root -associated fungal communities in relation to health status of Pisum sativum using barcoded pyrosequencing. Plant Soil. 2012;357:395-405.

27. Lemanczyk G, Sadowski CK. Fungal communities and health status of roots of winter wheat cultivated after oats and oats mixed with other crops. BioControl. 2002;47:349-61.

28. Yurgel SN, Abbey L, Loomer N, Gillis-Madden R, Mammoliti M. Microbial communities associated with storage onion. Phytobiomes J. 2018;2:35-41.

29. van Elsas JD, Chiurazzi M, Mallon CA, Elhottovā D, Krištůfek V, Salles JF. Microbial diversity determines the invasion of soil by a bacterial pathogen. Proc Natl Acad Sci. 2012:109:1159-64.

30. Schirmer M, ljaz UZ, D'Amore R, Hall N, Sloan WT, Quince C. Insight into biases and sequencing errors for amplicon sequencing with the Illumina MiSeq platform. Nucleic Acids Res. 2015;43:e37.

31. Ying-Wu S, Kai L, Chun L. Effects of endophytic fungus on sugar content and key enzymes activity in nitrogen and sugar metabolism of sugar beet (Beta vulgaris L.). Acta Agron Sin. 2009:35:946-51.

32. Atkins SD, Clark IM, Sosnowska D, Hirsch PR, Kerry BR. Detection and quantification of Plectosphaerella cucumerina, a potential biological control agent of potato cyst nematodes, by using conventional PCR, real-time PCR, selective media, and baiting. Appl Env Microbiol. 2003;69:4788-93.

33. Bailey K, Derby J-A, Bourdôt G, Skipp B, Cripps M, Hurrell G, et al. Plectosphaerella cucumerina as a bioherbicide for Cirsium arvense: proof of concept. BioControl. 2017:62:693-704.

34. Bulgarelli D, Rott M, Schlaeppi K, van Themaat EVL, Ahmadinejad N, Assenza F, et al. Revealing structure and assembly cues for Arabidopsis rootinhabiting bacterial microbiota. Nature. 2012;488:91-5.

35. Kolton M, Frenkel O, Elad Y, Cytryn E. Potential role of Flavobacterial gliding -motility and type IX secretion system complex in root colonization and plant defense. Mol Plant-Microbe Interact. 2014:27:1005-13.

36. Krishnamoorthy R, Kwon S-W, Kumutha K, Senthilkumar M, Ahmed S, Sa T, et al. Diversity of culturable methylotrophic bacteria in different genotypes of groundnut and their potential for plant growth promotion. 3 Biotech. 2018;8:275

37. Wei W, Zhou Y, Chen F, Yan X, Lai Y, Wei $C$, et al. Isolation, diversity, and antimicrobial and immunomodulatory activities of endophytic Actinobacteria from tea cultivars Zijuan and Yunkang-10 (Camellia sinensis var. assamica). Front Microbiol. 2018;9 https://doi.org/10.3389/fmicb.2018.01304.

38. Bugbee WM. Penicillium claviforme and Penicillium variabile: pathogens of stored sugarbeets. Phytopathology. 1975;65:926-7.

39. Snowdon AL. A colour atlas of post-harvest diseases and disorders of fruits and vegetables. Volume 1: General introduction and fruits. London: Wolfe Scientific Ltd., 1990.

40. Calabia BP, Tokiwa Y. Production of d-lactic acid from sugarcane molasses, sugarcane juice and sugar beet juice by Lactobacillus delbrueckii. Biotechnol Lett. 2007;29:1329-32.

41. du Preez JC, Bosch M, Prior BA. The fermentation of hexose and pentose sugars by Candida shehatae and Pichia stipitis. Appl Microbiol Biotechnol. 1986:23:228-33. 
42. Liebe S, Varrelmann M. Effect of environment and sugar beet genotype on root rot development and pathogen profile during storage.

Phytopathology. 2015;106:65-75.

43. Nirenberg H. Untersuchungen über die morphologische und biologische Differenzierung in der Fusarium-Sektion Liseola. Mitt Biol Bundesanst Land-U Forstwirtsch Eerlin-Dahlern. 1976;169:1-117.

44. Köljalg U, Nilsson RH, Abarenkov K, Tedersoo L, Taylor AFS, Bahram M, et al. Towards a unified paradigm for sequence-based identification of fungi. Mol Ecol. 2013;22:5271-7.

45. ICUMSA. Glucose and fructose in beet juices and processing products by an enzymatic method-accepted. Colney: Bartens; 2007.

46. ICUMSA. Polarisation of sugar beet by the macerator or cold aqueous digestion and aluminium sulphate. Colney: Bartens; 1994

47. Walters W, Hyde ER, Berg-Lyons D, Ackermann G, Humphrey G, Parada A, et al. Improved bacterial 165 rRNA gene (V4 and V4-5) and fungal internal transcribed spacer marker gene primers for microbial community surveys. mSystems. 2015;1 https://doi.org/10.1128/mSystems.00009-15.

48. Lundberg DS, Yourstone S, Mieczkowski P, Jones CD, Dangl JL. Practical innovations for high-throughput amplicon sequencing. Nat Methods. 2013; 10:999.

49. Caporaso JG, Kuczynski J, Stombaugh J, Bittinger K, Bushman FD, Costello EK, et al. QIIME allows analysis of high-throughput community sequencing data. Nat Methods. 2010;7:335-6.

50. Callahan BJ, McMurdie PJ, Rosen MJ, Han AW, Johnson AJA, Holmes SP. DADA2: high resolution sample inference from Illumina amplicon data. Nat Methods. 2016:13:581-3.

51. Quast C, Pruesse E, Yilmaz P, Gerken J, Schweer T, Yarza P, et al. The SILVA ribosomal RNA gene database project: improved data processing and webbased tools. Nucleic Acids Res. 2013;41:D590-6.

52. Nguyen NH, Song Z, Bates ST, Branco S, Tedersoo L, Menke J, et al. FUNGuild: an open annotation tool for parsing fungal community datasets by ecological guild. Fungal Ecol. 2016;20:241-8.

53. Ye J, Coulouris G, Zaretskaya I, Cutcutache I, Rozen S, Madden TL. PrimerBLAST: a tool to design target-specific primers for polymerase chain reaction. BMC Bioinformatics. 2012:13:134

54. Ogata K, Matsuda K, Tsuji H, Nomoto K. Sensitive and rapid RT-qPCR quantification of pathogenic Candida species in human blood. J Microbiol Methods. 2015;117:128-35.

55. Abd-Elsalam KA, Aly IN, Abdel-Satar MA, Khalil MS, Verreet JA. PCR identification of Fusarium genus based on nuclear ribosomal-DNA sequence data. Afr J Biotechnol. 2003;2:82-5.

56. Walter J, Hertel C, Tannock GW, Lis CM, Munro K, Hammes WP. Detection of Lactobacillus, Pediococcus, Leuconostoc, and Weissella species in human feces by using group-specific PCR primers and denaturing gradient gel electrophoresis. Appl Env Microbiol. 2001;67:2578-85.

57. Tannous J, Atoui A, El Khoury A, Kantar S, Chdid N, Oswald IP, et al. Development of a real-time PCR assay for Penicillium expansum quantification and patulin estimation in apples. Food Microbiol. 2015;50:28-37.

Ready to submit your research? Choose BMC and benefit from:

- fast, convenient online submission

- thorough peer review by experienced researchers in your field

- rapid publication on acceptance

- support for research data, including large and complex data types

- gold Open Access which fosters wider collaboration and increased citations

- maximum visibility for your research: over $100 \mathrm{M}$ website views per year

At $\mathrm{BMC}$, research is always in progress.

Learn more biomedcentral.com/submissions 